\title{
DISCONNECTED SOLUTIONS
}

\author{
BY W. F. LUCAS ${ }^{1}$ \\ Communicated by James H. Bramble, February 20, 1976
}

1. Introduction. In the book, Theory of games and economic behavior (1944), J. von Neumann and O. Morgenstern introduced a theory of solutions (or stable sets) for multi-person cooperative games in characteristic function form. A longstanding conjecture has been that the union of all solutions of any particular game is a connected set. (E.g., see [3].) This announcement describes a twelve-person game for which the conjecture fails. The essential definitions for an $n$-person game will be reviewed briefly before the counterexample is presented. A sketch of the proof is presented here, and the details will appear elsewhere.

2. The model. An n-person game is a pair $(N, v)$ where $N=\{1,2, \ldots, n\}$ is the set of players and $v$ is a characteristic function on $2^{N}$, i.e., $v$ assigns the real number $v(S)$ to each subset $S$ of $N$ and $v(\varnothing)=0$. The set of imputations is

$$
A=\left\{x: \sum_{i \in N} x_{i}=v(N) \text { and } x_{i} \geqslant v(\{i\}) \text { for all } i \in N\right\}
$$

where $x=\left(x_{1}, x_{2}, \ldots, x_{n}\right)$ is a vector with real components. For any $S \subset N$, let $x(S)=\Sigma_{i \in S} x_{i}$. For any $X \subset A$ and nonempty $S \subset N$, define $\operatorname{Dom}_{S} X$ to be the set of all $x \in A$ such that there exists a $y \in X$ with $y_{i}>x_{i}$ for all $i \in S$ and with $y(S) \leqslant v(S)$. Let Dom $X=\bigcup_{\phi \neq S \subset N} \operatorname{Dom}_{S} X$. A subset $V$ of $A$ is a solution if $V \cap \operatorname{Dom} V=\varnothing$ and $V \cup \operatorname{Dom} V=A$. The core of a game is

$$
C=\{x \in A: x(S) \geqslant v(S) \text { for all nonempty } S \subset N\} .
$$

For any solution $V, C \subset V$ and $V \cap \operatorname{Dom} C=\varnothing$.

A characteristic function $v$ is superadditive if $v(S \cup T) \geqslant v(S)+v(T)$ whenever $S \cap T=\varnothing$. The game below does not have a superadditive $v$ as is assumed in the classical theory, but it is equivalent solutionwise to a game with a superadditive $v$. (See $[1$, p. 68].)

3. Example. The 13 vital coalitions for our example consist of $N=$ $\{1,2,3,4,5,6,7,8,9,10,11,12\}$ and elements from three classes:

$B=\{\{1,2\},\{3,4\},\{5,6\},\{7,8\},\{9,10\},\{11,12\}\}$,

AMS (MOS) subject classifications (1970). Primary 90D12.

Key words and phrases. Game theory, solutions, stable sets, cores, characteristic functions, domination relations. 75-C-0678.

${ }^{1}$ Research supported in part by NSF grant MPS75-02024 and ONR contract N00014- 


$$
\begin{aligned}
& S=\{\{1,3,6,7,9,11\},\{1,4,5,7,9,11\},\{2,3,5,7,9,11\}\}, \\
& T=\{\{1,3,8\},\{1,5,10\},\{3,5,12\}\} .
\end{aligned}
$$

And $v$ is given by: $v(N)=6, v(S)=1$ for all $S \in B, v(S)=4$ for all $S \in S$, $v(S)=1$ for all $S \in T$, and $v(S)=0$ for all other $S \subset N$. For this game $A=$ $\left\{x: x(N)=6\right.$ and $x_{i} \geqslant 0$ for all $\left.i \in N\right\}$. Consider also the six-dimensional hypercube

$$
B=\{x \in A: x(S)=1 \text { for all } S \in B\}
$$

The core $C$ is the intersection of $C(S)$ and $C(T)$ where

$$
\begin{aligned}
& C(S)=\{x \in B: x(S) \geqslant 4 \text { for all } S \in S\}, \\
& C(T)=\{x \in B: x(S) \geqslant 1 \text { for all } S \in T\} .
\end{aligned}
$$

$C$ is a proper superset of the convex hull of the six vertices of $B$ which have $x_{i}=$ 1 for $i$ equal to five of the six odd indices $1,3,5,7,9$ and 11 , and $x_{i+1}=1$ when $i$ is the remaining odd numbered player. Let $\operatorname{Dom}_{B} X=\bigcup_{S \in B} \operatorname{Dom}_{S} X$. Note that $\operatorname{Dom}_{B} C \supset A-B$, and hence any-solution $V$ for our game is a subset of $B$.

4. Outline of proof. First, note that any component of an $x \in B$ has a maximum value of $x_{i}=1$. Consequently, the following three sets are contained in any solution $V$, i.e., they are subsets of $\bigcap V$ :

$$
\begin{aligned}
& E=\left\{x \in B: x_{i}=x_{j}=1 \text { for } i \neq j \text { and }\{i, j\} \subset\{1,3,5\}\right\}, \\
& F=\left\{x \in C(T): x_{p}=1 \text { for } p=7,9 \text { or } 11\right\}, \\
& P=\{(0,1,0,1,0,1,0,1,0,1,0,1)\} .
\end{aligned}
$$

Next, we can show that $\bigcup V$ must be a disconnected set. Let $G=\{x \in$ $B: x(\{7,9,11\}) \leqslant 1\}, G^{0}=\{x \in B: x(\{7,9,11\})<1\}$, and $P^{\prime}=\{x \in G:$ $\left.x_{2}=x_{4}=x_{6}=1\right\}$. Throughout this section the indices $i, j$ and $k$ represent some ordering of the distinct indices 1, 3 and 5. The subset $H$ of $E$ consisting of the three triangular regions

$$
H_{i}=\left\{x \in G: x_{i+1}=x_{j}=x_{k}=1 ; x_{7}+x_{9}+x_{11}=1\right\}
$$

is in $\bigcap V$ and $\operatorname{Dom}_{S} N \supset G^{0}-\left(E \cup P^{\prime}\right)$. The subset $J$ of $F$ consisting of the three triangular regions

$$
\begin{aligned}
& J_{1}=\left\{x \in F: x_{1}=x_{7}=x_{9}=1, x_{3}+x_{5}+x_{12}=1\right\}, \\
& J_{3}=\left\{x \in F: x_{3}=x_{7}=x_{11}=1, x_{1}+x_{5}+x_{10}=1\right\}, \\
& J_{5}=\left\{x \in F: x_{5}=x_{9}=x_{11}=1, x_{1}+x_{3}+x_{8}=1\right\}
\end{aligned}
$$

is also in $\cap V$ and $\operatorname{Dom}_{T} J \supset B-C(T) \supset P^{\prime}-P$. So any $x \in \cup V-P$ either has $x \in E$ or $x \in B-G^{0}$, i.e., $x_{i}=x_{j}=1$ or $x(\{7,9,11\}) \geqslant 1$. Such $x$ are clearly disconnected from the singleton $P \subset \cap V$.

Finally, it is necessary to demonstrate that this game does possess at least one solution. $V^{\prime}=C \cup E \cup F \cup P$ is in any solution $V$, and $V^{\prime}$ can be enlarged to a solution in two steps. First, include the set of imputations $L$ in $C(T)-$ 
$\left(V^{\prime} \cup\right.$ Dom $\left.V^{\prime}\right)$ which is simultaneously maximal with respect to all three of the relations "Dom $s$ " for $S \in S$. Clearly $L \subset \bigcap V$. Next, pick a particular $S^{i}=$ $\{i+1, j, k, 7,9,11\} \in S$ and then add in those elements $L^{i}$ in $C(T)-\left(V^{\prime} \cup\right.$ $L \cup \operatorname{Dom}\left(V^{\prime} \cup L\right)$ ) which are maximal with respect to the relation "Dom $S^{i}$ " and are at the same time symmetrical in the sense that $x_{j}=x_{k}$. It requires some detail to describe the sets $L$ and $L^{i}$ explicitly, and to verify that the resulting sets $V^{i}=V^{\prime} \cup L \cup L^{i}$ are solutions for our example. These will appear elsewhere

5. Remarks. At one time it was apparently believed that proving the union of all solutions connected could be a major step in showing that every game has a solution. It is now known [2] that a solution need not exist for every game. On the other hand, it is possible that results on disconnecting $U V$ might be useful in the resolution of important open questions about whether solutions always exist for games with full-dimensional cores, with empty cores, or which are constant-sum.

\section{REFERENCES}

1. D. B. Gillies, Solutions to general non-zero-sum games, Ann. of Math. Studies, no. 40 (A. W. Tucker and R. D. Luce, eds.), Princeton Univ. Press, Princeton, N.J., 1959, pp. 47-85. MR $21 \# 4850$.

2. W. F Lucas, The proof that a game may not have a solution, Trans. Amer. Math. Soc. 137 (1969), 219-229. MR $38 \# 5474$.

3. L. S. Shapley, Open questions, Report of an Informal Conf. on the Theory of n-Person Games (Princeton Univ., March 20-21, 1953), H. W. Kuhn, ed., Princeton, Univ. Press, Princeton, N.J., p. 15.

SCHOOL OF OPERATIONS RESEARCH AND CENTER FOR APPLIED MATHEMATICS, CORNELL UNIVERSITY, ITHACA, NEW YORK 14853 